\title{
DGN-Jahresberichte 2019
}

Die folgenden Tabellen stellen die Aktivitäten der DGN-Ausschüssse ( $\triangleright$ Tab. 1) und Arbeitsgemeinschaften $(\triangleright$ Tab. 2) sowie der Regionalgesellschaften ( $\triangleright$ Tab. 3) im Berichtszeitraum 2019 dar.

- Tab. 1 Jahresberichte 2019: DGN-Ausschüsse

\begin{tabular}{|c|c|c|}
\hline $\begin{array}{l}\text { Ausschuss, } \\
\text { Vorsitz }\end{array}$ & \multicolumn{2}{|c|}{ Zusammenkünfte, Aktivitäten im Berichtszeitraum und Planungen } \\
\hline \multirow{2}{*}{$\begin{array}{l}\text { Ethik } \\
\text { Prof. W. H. Knapp }\end{array}$} & Veranstaltungen & \\
\hline & Aktivitäten & $\begin{array}{l}\text { - Erstellung einer Erklärung zur Selbstverpflichtung der DGN zu Transparenz, Ethik und Vermeidung von } \\
\text { Interessenkonflikten } \\
\text { - Ausschuss-Sitzung, 03. April 2019, Bremen } \\
\text { - Diskussion der Bewertungsgrundlagen für eingegangene Interessenerklärungen } \\
\text { - Präzisierung der Verfahrensregelungen innerhalb des Ausschusses und mit der Geschäftsstelle } \\
\text { - Telefonkonferenz, 26. April } 2019 \\
\text { - Konsensfindung und abschließende Bewertung der Interessenerklärungen der DGN-Amtsträger durch } \\
\text { den Ausschuss Ethik } \\
\text { - Vorschlag an DGN-Vorstand zur Offenlegung der sekundären Interessen und deren Bewertung im } \\
\text { Mitgliederbereich }\end{array}$ \\
\hline \multirow[t]{3}{*}{$\begin{array}{l}\text { Fort- und } \\
\text { Weiterbildung } \\
\text { Prof. W. Brenner }\end{array}$} & Veranstaltungen & $\begin{array}{l}\text { - Ausschuss-Sitzung im Rahmen der DGN-Jahrestagung } 2019 \text { in Bremen } \\
\text { - DGN-Summer School: „Translational Research in Molecular Imaging and Radionuclide Therapy“, } \\
\text { 29.-31. August 2019, Berlin } \\
\text { - Monatlich Telefonkonferenzen }\end{array}$ \\
\hline & Aktivitäten & $\begin{array}{l}\text { - Fort- und Weiterbildungsaktivitäten } \\
\text { - Organisation CME-Sessions der Jahrestagungen } 2019 \text { und } 2020 \\
\text { - Beteiligung an DGN-Veranstaltungen oder anderen Veranstaltungen } \\
\text { - DGN-Summer School } 2019 \\
\text { - Vorbereitung der DGN-Summer School } 2020 \\
\text { - Vorbereitung der CME-Sessions der DGN-Jahrestagung } 2020 \\
\text { - Teilnahme UEMS/EBNM National Delegates Assembly während der EANM } 2019 \text { in Barcelona } \\
\text { - Zusammenarbeit mit DGN-Gremien } \\
\text { - Konzepterstellung einer „Fachakademie Nuklearmedizin“ mit dem Vorstand } \\
\text { - Zusammenarbeit mit anderen Gremien/Kommissionen } \\
\text { - CME-Sessions der DGN-Jahrestagung je nach Thema in Abstimmung mit dem jeweils zuständigen } \\
\text { - Ausschuss } \\
\text { - anderweitige Aktivitäten (z. B. Studien, Publikationen) } \\
\text { - Organisation Mitarbeit Pschyrembel: Fachbereich Nuklearmedizin und zugehörige Physik, Medizintechnik } \\
\text { - Pund Radiopharmazie für Online-Ausgabe 2019 } \\
\quad \text { Nuklearmedizin 2019 Oct 10. doi:10.1055/a-1011-3481 [Epub ahead of print] }\end{array}$ \\
\hline & Vorhaben & $\begin{array}{l}\text { - DGN-Summer School 2020, 27.-29. August 2020, Berlin } \\
\text { - Ausschusssitzungen im Rahmen der DGN-Jahrestagung } 2020 \text { sowie regelmäßige Telefonkonferenzen } \\
\text { - IMPP-Fragen zur Nuklearmedizin aktualisieren } \\
\text { - Begleitung Musterweiterbildungsordnung } \\
\text { - Konzept und Etablierung eines Lehrpreises ab } 2021 \\
\text { - Abklärung Möglichkeiten E-Learning im Rahmen der DGN }\end{array}$ \\
\hline \multirow{2}{*}{$\begin{array}{l}\text { Leistungserfassung } \\
\text { und -vergütung } \\
\text { PD M. Freesmeyer }\end{array}$} & Veranstaltungen & $\begin{array}{l}\text { - Ausschusssitzung, 03. April 2019, Bremen } \\
\text { - Telefonkonferenz, 30. September } 2019\end{array}$ \\
\hline & Aktivitäten & $\begin{array}{l}\text { - Fort- und Weiterbildungsaktivitäten } \\
\text { - Evaluation einer Fortbildungsveranstaltung „Kodier-Workshop“ } \\
\text { - die Nuklearmedizin im DRG-System } 2019 \text { und Ausblick auf 2020; Kalkulation, Erlöse, Budgets und der } \\
\text { „Pflexit“ } \\
\text { - Zusammenarbeit mit DGN-Gremien } \\
\text { - Ausschuss Fort- und Weiterbildung } \\
\text { - Zusammenarbeit mit anderen Fachgesellschaften } \\
\text { - Deutsche Gesellschaft für Urologie } \\
\text { - Deutsche Gesellschaft für Interventionelle Radiologie } \\
\text { - Zusammenarbeit mit anderen Gremien/Kommissionen } \\
\text { - DIMDI }\end{array}$ \\
\hline
\end{tabular}


Tab. 1 (Fortsetzung)

\section{Ausschuss, $\quad$ Zusammenkünfte, Aktivitäten im Berichtszeitraum und Planungen \\ Vorsitz}

- anderweitige Aktivitäten (z. B. Studien, Publikationen)

- erfolgreiche Antragstellung für OPS-Änderung

- Tc-Tilmanocept (3-709.01)

- Lutathera (8-530.62)

- Tumorszintigrafie (3-70c.0)

- Holmium - Scout-Dose (3-708.01)

- Holmium - intratherapeutische Dosimetrie (8-539.03)

- Antragstellung

- ZE-Antrag Lutathera

- Holmium-SIRT inkl. Scout-Dose

- Verteilung eines Fragebogens, der deutschlandweit Zusatz- und NUB-Entgelte erfasst (Dezember 2019)

- Neuerstellung der Kodierempfehlungen gemäß des DRG- und OPS-Katalogs 2019 sowie Distribution an die deutschlandweiten Kalkulationskrankenhäuser via Geschäftsstelle mit dem Ziel der einheitlichen und korrekten Kodierung

- Vorbereitung OPS-Änderungen

- 8-530.60 (Abrechnung Lutathera)

- 8-530.60/3-754 (Öffnung für weitere Chelatoren außer DOTA)

- 3-70c.3 (Tumorszintigrafie mit Tc-PSMA) in Verbindung mit Radioguided Surgery

- 3-709.0 (Tilmanocept)

\begin{tabular}{l|ll} 
Vorhaben & - Beantragung o.g. OPS-Änderungen
\end{tabular}

- aktive Prüfung des Sachkostenanteils M10B (Lu-PSMA-Therapie), Erarbeitung einer spezifischen Kodierempfehlung und Distribution an die Kalkulationskrankenhäuser, um eine Abwertung 2020 zu verhindern

- Auswertung des Fragebogens, der Zusatz- und NUB-Entgelte erfasst

- Überarbeitung und Veröffentlichung der Kodierrichtlinien für 2020

- Erarbeitung einer OPS-Änderung 8-530.d0 analog 8-530.60, wenn zugelassenes Lu-PSMA zur Verfügung steht

- Werbung Zentralisierung NUB-Anträge

- Vorschlag an Vorstand, einen externen Berater zur Optimierung der Vergütungssituation Lu-PSMA nach Zulassung zu engagieren

\section{Leitlinien}

Prof. M. Schmidt

Veranstaltungen

- Sitzung des Arbeitsausschusses Leitlinien im Rahmen der DGN-Jahrestagung, 03. April 2019, Bremen

- Treffen der Steuergruppe beim Henning-Symposium in Heidelberg,

11. Oktober 2019, Heidelberg

- 20. Jahrestagung der Selbsthilfegruppe Schilddrüsenkarzinom,

26. Oktober 2019, Berlin

- S3-Leitlinienprojekt „Schilddrüsenkarzinom“, Köln, Essen, Göttingen, Berlin

Aktivitäten $\quad$ " Leitlinien

- Aktualisierung der LL zur mIBG-Szintigrafie beim Neuroblastom und zur Nebenschilddrüsenszintigrafie

- weitere LL-Aktualisierungen, e. g. Hirnperfusions-SPECT mit Tc-99m-Radiopharmaka, Radioimmuntherapie des CD20-positiven follikulären B-Zell-Non-Hodgkin-Lymphoms, Nierenszintigrafie bei Kindern

- Fort- und Weiterbildungsaktivitäten

- 26. Hamburger Nuklearmedizin-Tage 05.-07. Juni 2019

- Beteiligung an DGN-Veranstaltungen oder anderen Veranstaltungen

- Einladung der Alexander-von-Humboldt-Stiftung zur Konferenz „Atoms for Peace“ vom 14.-15. Oktober 2019

- Zusammenarbeit mit DGN-Gremien

- DGN-Ausschuss „Fort- und Weiterbildung“, DGN-Ausschuss „Ethik“, Zusammenarbeit mit dem Therapieund PET-Ausschuss

- Zusammenarbeit mit anderen Fachgesellschaften

- European Neuroendocrine Tumor Society

- Deutsche Gesellschaft für Endokrinologie

- anderweitige Aktivitäten (z. B. Studien, Publikationen)

- Publikation: Bedeutung und Entwicklung von Leitlinien seitens der Deutschen Gesellschaft für Nuklearmedizin. Der Nuklearmediziner 2019; 42: 283-290

Vorhaben _ - S3-Leitlinie „Schilddrüsenkarzinom“ im Jahr 2020

- Aktualisierung der SLN-LL

Medizinphysik

PD Dr. S. Nekolla
Veranstaltungen
- CME-Curriculum „Quantifizierung“ im Rahmen von Refresher-Kursen während der DGN-Jahrestagung. Dieses Curriculum ist auf 4 Jahre angelegt. Auf der DGN-Jahrestagung wurde Teil 1 „Quantifizierung in der nuklearmedizinischen Bildgebung I: messtechnische Aspekte, Korrekturen und quantitative Größen“ durchgeführt und wurde sehr positiv aufgenommen.

- Ausschusstreffen, 04. April 2019, Bremen

- Telefonkonferenz, 11. Dezember 2019 
- Tab. 1 (Fortsetzung)

\section{Ausschuss, Zusammenkünfte, Aktivitäten im Berichtszeitraum und Planungen \\ Vorsitz}

\begin{tabular}{|c|c|c|}
\hline & Aktivitäten & $\begin{array}{l}\text { - Fort- und Weiterbildungsaktivitäten } \\
\text { - Mitarbeit an der Definition von Kenntnissen und Tätigkeiten von Medizinphysik-Experten } \\
\text { - Beteiligung an DGN-Veranstaltungen oder anderen Veranstaltungen } \\
\text { - GAPTN, Hannover } \\
\text { - Zusammenarbeit mit DGN-Gremien } \\
\text { - Ausschuss für Dosimetrie } \\
\text { - Ausschuss Strahlenschutz } \\
\text { - Ausschuss Therapie } \\
\text { - Zusammenarbeit mit anderen Fachgesellschaften } \\
\text { - EANM Dosimetry Committee } \\
\text { - EANM Physics Committee } \\
\text { - EANM Radiation Protection Committee } \\
\text { - DGMP (GAPTN) }\end{array}$ \\
\hline & Vorhaben & $\begin{array}{l}\text { - Aufbau einer Infrastruktur für eine webbasierte Artefakt-Datenbank durch die Klinik für Nuklearmedizin der } \\
\text { Universität Leipzig (technische Unterstützung weiterhin erforderlich } \rightarrow \text { derzeit Testphase auf proprietärer } \\
\text { Infrastruktur, perspektivisch Providing durch DGN/vokativ). Evaluierung durch Ausschussmitglieder in } 2019 . \\
\text { Vorstellung des Status auf DGMP-Winterschule in Pichl (März 2019), dann Erweiterung des Kreises der Test- } \\
\text { benutzer, derzeit weitere Revision und Verbesserung der Plattform. Akzeptanz noch nicht im erwünschten } \\
\text { Bereich, liegt aber auch an noch nicht ausreichender Werbung (bisher keine Rückäußerung von Vokativ nach } \\
\text { Gespräch BS mit Herrn Jonas auf DGN } 2019 \text { in Bremen). } \\
\text { - im Rahmen des CME-Curriculums „Quantifizierung“ wird während der DGN-Jahrestagung } 2020 \text { ein } \\
\text { weiteres Modul angeboten } \\
\text { - 2. Quantifizierung in der Therapie I: Grundlagen durchgeführt. Die Themen umfassen physikalische Prinzipien } \\
\text { und Grundlagen der nuklearmedizinischen Therapie, Inkorporationsdosimetrie diagnostischer Radiopharma- } \\
\text { zeutika, Dosimetrie für Radionuklidtherapien - die Grundlagen und praktische Dosimetrie in der Therapie am } \\
\text { Beispiel ausgewählter kommerzieller Lösungen. }\end{array}$ \\
\hline \multirow[t]{2}{*}{$\begin{array}{l}\text { PET } \\
\text { Prof. C. Lapa }\end{array}$} & Aktivitäten & $\begin{array}{l}\text { - eigene Veranstaltungen } \\
\text { - Prüfung PET-Zertifikate bei DGN-Jahrestagung } \\
\text { - Zusammenarbeit mit DGN-Gremien } \\
\text { - Vorstand } \\
\text { - Ausschüsse Leitlinien, Multicenterstudien, Leistungserfassung und -vergütung } \\
\text { - anderweitige Aktivitäten (z. B. Studien, Publikationen) } \\
\text { - Beantwortung von PET-Fragen an den „Expertenmakler“ auf der DGN-Homepage }\end{array}$ \\
\hline & Vorhaben & $\begin{array}{l}\text { - Sitzung im Rahmen der DGN-Jahrestagung, April 2020, Leipzig } \\
\text { - Update Leitlinie „PET“ (bis Ende 2020) } \\
\text { - Definition und Zertifizierung PET-Zentrum (bis Ende 2020) } \\
\text { - Aktualisierung/Neugestaltung PET-Zertifikat } \\
\text { - Planung/Initiierung Multicenterstudie (z. B. PSMA-PET/CT) aufbauend auf HTA-Bericht }\end{array}$ \\
\hline \multirow[t]{2}{*}{$\begin{array}{l}\text { Radiopharmaka } \\
\text { Prof. T. Kuwert }\end{array}$} & Veranstaltungen & $\begin{array}{l}\text { - Ausschusssitzung } \\
\text { - DGN-Jahrestagung, Bremen, 03. April } 2019 \\
\text { - AGRR-Jahrestagung, Pamhagen, 12. September } 2019\end{array}$ \\
\hline & Aktivitäten & $\begin{array}{l}\text { - Fort- und Weiterbildungsaktivitäten } \\
\text { - CME-Fortbildung auf dem DGN-Kongress in Bremen: Fortbildung } 7 \text { „Radiochemie für Mediziner: Von der } \\
\text { Gesetzeslage zur Tracerentwicklung“ } \\
\text { Referent/Thema: } \\
\text { Dr. Franz Gildehaus: „Radiochemie“ } \\
\text { Dr. Christoph Solbach: „Gesetzeslage Radiopharmaka“ } \\
\text { Prof. Dr. Torsten Kuwert: „Tracerentwicklung“ } \\
\text { - GMP-Weiterbildung im Vorfeld der AGGR-Tagung } \\
\text { (12.-14. September 2019, Pamhagen) } \\
\text { Referent/Thema: } \\
\text { Dr. E. Pel, EDQM: „Das Europäische Arzneibuch“ } \\
\text { Mag. A. Kraßnigg, BASG/AGES: „Die Rolle der sachkundigen Person” } \\
\text { - Beteiligung an DGN-Veranstaltungen oder anderen Veranstaltungen } \\
\text { - Jahrestagung DGN } \\
\text { - Jahrestagung AGRR } \\
\text { - eigene Veranstaltungen } \\
\text { - GMP-Schulung im Vorfeld der AGGR-Tagung, 12.-14. September 2019, Pamhagen (s. o.) }\end{array}$ \\
\hline
\end{tabular}


- Tab. 1 (Fortsetzung)

\section{Ausschuss, $\quad$ Zusammenkünfte, Aktivitäten im Berichtszeitraum und Planungen \\ Vorsitz}

- Zusammenarbeit mit DGN-Gremien

- DGN-Vorstand und -Geschäftsstelle

- AGRR

- Telefonkonferenzen

- Zusammenarbeit mit anderen Fachgesellschaften

- EANM

- AGRR

- DPhG

- Zusammenarbeit mit anderen Gremien/Kommissionen

- EFG12 ZLG

- Arzneibuchkommission BfArM

- Radiopharmacy Committee EANM

- Expert Group 14 EDQM, European Pharmacopoeia

\begin{tabular}{|c|c|c|}
\hline & & - Expert Group 14 EDQM, European Pharmacopoeia \\
\hline & Vorhaben & $\begin{array}{l}\text { - Mediziner-Weiterbildung inkl. Podiumsdiskussion anlässlich DGN-Jahrestagung, April } 2020 \\
\text { - GMP-Schulung anlässlich AGRR-Jahrestagung, September } 2020 \\
\text { - Ausschusssitzung anlässlich DGN-Jahrestagung, April } 2020 \\
\text { - Ausschusssitzung anlässlich AGRR-Jahrestagung, September } 2020 \\
\text { - Projekt „Hotline Radiopharmaka“, 2020/2021 } \\
\text { - Verständigung EFG der ZLG } 12 \text { (Vorsitzende Frau Karmann, Reg. Oberbayern), } 2020 \\
\text { - Unterstützung Initiative „Öffnung AMRadV für Radiotherapeutika“, } 2020 \\
\text { - Unterstützung Projekt „Definition Therapiezentren“, } 2020 \\
\text { - Klärung der Rechtssituation für Radiopharmaka, die rein zu Forschungszwecken bei Probanden eingesetzt } \\
\text { - } \text { werden, } 2020\end{array}$ \\
\hline \multirow{3}{*}{$\begin{array}{l}\text { Schilddrüse } \\
\text { Prof. M. KreißI }\end{array}$} & Veranstaltungen & - Ausschuss-Sitzung im Rahmen der DGN-Jahrestagung, 04. April 2019, Bremen \\
\hline & Aktivitäten & $\begin{array}{l}\text { - Leitlinien } \\
\text { - S3-Leitlinie „Schilddrüse“ } \\
\text { - diagnostische Ganzkörperszintigrafie, Version } 5 \\
\text { - Mitwirkung: Consensus statement and recommendation - Non-surgical and non-radioiodine techniques } \\
\text { for ablation of benign thyroid nodules (mit Sektion Schilddrüse der DGE und CAEK) } \\
\text { - Beteiligung an DGN-Veranstaltungen oder anderen Veranstaltungen } \\
\text { - CME-Session, DGN-Jahrestagung 2019, Bremen } \\
\text { - „Heidelberger Schilddrüsensymposium“, Oktober } 2019 \\
\text { - Tagung der Sektion Schilddrüse der DGE, November } 2019 \\
\text { - Festsymposium „20 Jahre Selbsthilfe Schilddrüsenkrebs”, 26. Oktober } 2019 \\
\text { - Zusammenarbeit mit DGN-Gremien } \\
\text { - Ausschuss PET } \\
\text { - Ausschuss Leitlinie } \\
\text { - Zusammenarbeit mit anderen Fachgesellschaften } \\
\text { - DGE (Sektion Schilddrüse) } \\
\text { - CAEK } \\
\text { - Thyroid committee, EANM } \\
\text { - Zusammenarbeit mit anderen Gremien/Kommissionen } \\
\text { - Selbsthilfegruppe Schilddrüsenkrebs } \\
\text { " anderweitige Aktivitäten (z. B. Studien, Publikationen) } \\
\text { - Erarbeitung Statement zu Kitahara et al. 2019 (laufend) } \\
\text { - Erstellung Statement zur Nutzenbewertung von Lenvima }{ }^{\circledR} \text { beim fortgeschrittenen Schilddrüsenkarzinom } \\
\text { beim G-BA (koordiniert mit Sektion Schilddrüse der DGE und CAEK) } \\
\text { - Mitwirkung an Neufassung für das Kapitel Stoffwechsel, innere Sekretion beim Bundesministerium für } \\
\text { Arbeit und Soziales (schilddrüsenbezogen) zur Festlegung GdB/GdS }\end{array}$ \\
\hline & Vorhaben & $\begin{array}{l}\text { - CME-Session, DGN-Jahrestagung 2020, Leipzig } \\
\text { - Tagung des Ausschusses Schilddrüse, Herbst } 2020\end{array}$ \\
\hline $\begin{array}{l}\text { Strahlenschutz } \\
\text { Prof. W. Burchert }\end{array}$ & Veranstaltungen & $\begin{array}{l}\text { - Treffen des Ausschusses Strahlenschutz im Rahmen der DGN-Jahrestagung 2019, 03. April 2019, Bremen } \\
\text { - Vorstellung neuer Ausschuss Strahlenschutz; Abgrenzung zum Ausschuss Dosimetrie; Ausschuss } \\
\text { Medizinische Physik } \\
\text { - Orientierungshilfe bildgebende Untersuchungen SSK } \\
\text { - Entsorgung: langlebige Nuklide in Diagnostika etc. } \\
\text { - Risikoanalyse allgemeines Verfahren erarbeiten? }\end{array}$ \\
\hline
\end{tabular}


- Tab. 1 (Fortsetzung)

\section{Ausschuss, Zusammenkünfte, Aktivitäten im Berichtszeitraum und Planungen \\ Vorsitz}

- Diskussion Prüfverfahren auf Heilkunde einstellen? Empfehlung für Vorstand; Fachgespräch beim BFS

- kommende Richtlinien; Fachkunderichtlinie (Burchert)

- Kapitel Leitlinien?

- Fortbildung „Neues Strahlenschutzrecht“

- DIN-NAR AA3 Nuklearmedizin - Änderungen in der Trägerschaft

\begin{tabular}{|c|c|c|}
\hline & \multirow[b]{2}{*}{ Aktivitäten } & \\
\hline & & $\begin{array}{l}\text { - Leitlinien, Gesetze } \\
\text { - Fachkunderichtlinie MPE } \\
\text { - Orientierungsleitfaden Bildgebende Verfahren (SSK) } \\
\text { - Referentenentwurf der Allgemeinen Verwaltungsvorschrift zur Ermittlung der Exposition von } \\
\text { Einzelpersonen in der Bevölkerung durch genehmigungs- und anzeigebedürftige Tätigkeiten } \\
\text { - Beteiligung an DGN-Veranstaltungen oder anderen Veranstaltungen } \\
\text { - Treffen des Ausschusses Strahlenschutz im Rahmen der DGN Jahrestagung 2019, 03. April 2019, Bremen } \\
\text { (s. o.) } \\
\text { - BfS-Konsultationstreffen: Medizinische Forschung, 24. Mai 2019, BfS Oberschleißheim } \\
\text { - eigene Veranstaltungen } \\
\text { - s. u. Veranstaltungen } \\
\text { - Zusammenarbeit mit DGN-Gremien } \\
\text { - AG GAPTN } \\
\text { - Ausschuss Leitlinien } \\
\text { - Ausschuss Therapie } \\
\text { - Vorstand DGN } \\
\text { - Ausschuss Dosimetrie } \\
\text { - Ausschuss Medizinische Physik } \\
\text { - Zusammenarbeit mit anderen Fachgesellschaften } \\
\text { - EANM Dosimetry Committee } \\
\text { - EANM Physics Committee } \\
\text { - DEGRO } \\
\text { - DRG } \\
\text { - DGMP } \\
\text { - Zusammenarbeit mit anderen Gremien/Kommissionen } \\
\text { - Strahlenschutzkommission (SSK) } \\
\text { - Normenausschuss Radiologie (NAR des Deutschen Instituts für Normung e. V. (DIN)) } \\
\text { - Bundesamt für Strahlenschutz (u. a. Fachgespräche, Gesetzesvorbereitung) } \\
\text { - Bundesministerium für Umwelt, Naturschutz und nukleare Sicherheit (BMUB) } \\
\text { - ZAES } \\
\text { - anderweitige Aktivitäten (z. B. Studien, Publikationen) } \\
\text { - Stellungnahmen zu G-BA-Beschlüssen } \\
\text { - Teilnahme an G-BA-Anhörungen }\end{array}$ \\
\hline & Vorhaben & $\begin{array}{l}\text { - Übersetzung Orientierungsleitfaden } \\
\text { - Richtlinie Strahlenschutz in der Medizin, Fachkunderichtlinien } \\
\text { - Überführung NAR in das DIN }\end{array}$ \\
\hline \multirow[t]{3}{*}{$\begin{array}{l}\text { Therapie } \\
\text { Prof. K. Rahbar }\end{array}$} & Veranstaltungen & $\begin{array}{l}\text { - Sitzung im Rahmen der DGN-Jahrestagung, 03. April 2019, Bremen } \\
\text { - Strategietreffen der DGN, 22. November } 2019 \text { (Rahbar) } \\
\text { - Hochschulausschuss, 22. November } 2019 \text { (Rahbar) }\end{array}$ \\
\hline & Aktivitäten & $\begin{array}{l}\text { - Leitlinien (u. a. Aktualisierungen) } \\
\text { - selektive intraarterielle Radiotherapie (SIRT) maligner Lebertumoren } \\
\text { - Radionuklidtherapie von Knochenmetastasen mittels Radium-223 } \\
\text { - Peptidrezeptor-Radionuklidtherapie } \\
\text { - Zusammenarbeit mit DGN-Gremien } \\
\text { - Ausschuss Leitlinien } \\
\text { - Ausschuss Dosimetrie } \\
\text { - Aktivitäten } \\
\text { - Bildung von DGN-Therapiezentren } \\
\text { - Informationsplattform Therapien in Deutschland }\end{array}$ \\
\hline & Vorhaben & $\begin{array}{l}\text { - Bildung von DGN-Therapiezentren (Etablierung) } \\
\text { - DGN-Kurs/Workshop zur Radionuklidtherapie, Inhalte: Therapieprozeduren, Medizinphysik inkl. } \\
\text { Dosimetrie, Strahlenschutz, Onkologie (Grundlagen Toxizität und Handling), Palliativmedizin }\end{array}$ \\
\hline
\end{tabular}


- Tab. 2 Jahresberichte 2019: DGN-Arbeitsgemeinschaften

\begin{tabular}{|c|c|c|}
\hline $\begin{array}{l}\text { Arbeitsgemeinschaft, } \\
\text { Vorsitz }\end{array}$ & Zusammenkünft & ktivitäten im Berichtszeitraum und Planungen \\
\hline \multirow{2}{*}{$\begin{array}{l}\text { In-vitro-Diagnostik } \\
\text { PD. Dr. M. Zimny }\end{array}$} & Veranstaltungen & - Sitzungstreffen im Rahmen der DGN-Jahrestagung, 05. April 2019 \\
\hline & Aktivitäten & $\begin{array}{l}\text { - Fort- und Weiterbildungsaktivitäten } \\
\text { - Fortbildungssitzung DGN-Jahrestagung Bremen } \\
\text { - Fortbildungsvortrag Jahrestagung BDN 09/19 Berlin } \\
\text { - Fortbildungsvortrag QZ Nuklearmedizin 09/19 Wiesbaden } \\
\text { - Beteiligung an DGN-Veranstaltungen oder anderen Veranstaltungen } \\
\text { - DGN-Jahrestagung in Bremen } \\
\text { - BDN-Jahrestagung in Berlin }\end{array}$ \\
\hline \multirow{3}{*}{$\begin{array}{l}\text { Kardiovaskuläre } \\
\text { Nuklearmedizin und } \\
\text { Lungendiagnostik } \\
\text { der DGN } \\
\text { Prof. W. Schäfer }\end{array}$} & Veranstaltungen & $\begin{array}{l}\text { - Treffen im Rahmen der DGN-Jahrestagung, 04. April 2019, Bremen } \\
\text { - Nuklearkardiologie-Workshop bei den Kardiodiagnostiktagen 2019, 21.-23. Februar 2019, } \\
\text { Lindner/Bucerius }\end{array}$ \\
\hline & Aktivitäten & $\begin{array}{l}\text { - Fort- und Weiterbildungsaktivitäten } \\
\text { - siehe unten: 14. Kurs kardiovaskuläre Nuklearmedizin/Nuklearkardiologie Potsdam, u. a. Falldemos } \\
\text { und -diskussionen, Erläuterung der neuen ESC-Guidelines } \\
\text { - Workshop und Vorträge im Rahmen der Kardiodiagnostiktage } 2019 \\
\text { - Beteiligung an DGN-Veranstaltungen oder anderen Veranstaltungen } \\
\text { - Sitzung der Arbeitsgruppe „Nuklearkardiologische Diagnostik“ im Rahmen der DGK, 24. April 2019, } \\
\text { Mannheim } \\
\text { - eigene Veranstaltungen } \\
\text { - 14. Kurs für kardiovaskuläre Nuklearmedizin/Nuklearkardiologie, } \\
\text { 12.-14. September 2019, Potsdam } \\
\text { - Zusammenarbeit mit anderen Fachgesellschaften } \\
\text { - DGK } \\
\text { - BDN } \\
\text { - EANM } \\
\text { - Zusammenarbeit mit anderen Gremien/Kommissionen } \\
\text { - Mitglied des Cardiovascular Committee der EANM } \\
\text { - Organisation der ESMIT-Summer School in Zagreb } \\
\text { - Gestaltung von webbasierten Lehrmedien } \\
\text { - anderweitige Aktivitäten (z. B. Studien, Publikationen) } \\
\text { - 8. Umfrage zur Myokard-SPECT (Start Januar 2019) } \\
\text { - Publikation zur } 8 . \text { Umfrage zur Myokard-SPECT } \\
\text { - Ausgabe Januar } 2020 \text { von „Der Nuklearmediziner“ (Thieme-Verlag) zum Thema „Nuklearkardiologie“ }\end{array}$ \\
\hline & Vorhaben & - 1. Umfrage zur Lungen-SPECT (Start Januar 2020) \\
\hline \multirow[t]{2}{*}{$\begin{array}{l}\text { MTM } \\
\text { R. Höhne }\end{array}$} & Veranstaltungen & $\begin{array}{l}\text { - Sitzung im Rahmen der DGN-Jahrestagung, 05. April 2019, Bremen } \\
\text { " Mitgliederversammlung im Rahmen der DGN-Jahrestagung, 05. April 2019, Bremen } \\
\text { " Vorstandssitzung, 13./14. Juli 2019, Heidelberg } \\
\text { - Diverse Telefonkonferenzen }\end{array}$ \\
\hline & Aktivitäten & $\begin{array}{l}\text { - Beteiligung an DGN-Veranstaltungen oder anderen Veranstaltungen } \\
\text { - Organisation der MTRA-Tagung im Rahmen der 57. Jahrestagung der Deutschen Gesellschaft } \\
\text { für Nuklearmedizin in Bremen } \\
\text { - Vortrag von R. Höhne zum „Stellenwert nuklearmedizinischer Methoden“, 11. Deutsche } \\
\text { Kardiodiagnostiktage, 23. Februar 2019, Leipzig } \\
\text { - Vortrag von S. Biedenstein zur „Tumortherapie in der Nuklearmedizin“, 25. DEGRO-Jahrestagung, } \\
\text { 14. Juni 2019, Münster } \\
\text { - eigene Veranstaltungen } \\
\text { - MTRA-Tagung im Rahmen der 57. Jahrestagung der Deutschen Gesellschaft für Nuklearmedizin } \\
\text { in Bremen } \\
\text { - Zusammenarbeit mit DGN-Gremien } \\
\text { - Stellungnahme zum Positionspapier des VMTB zur Thematik des Fachkräftemangels mit dem Vorstand } \\
\text { der DGN } \\
\text { - Zusammenarbeit mit anderen Fachgesellschaften } \\
\text { - die inzwischen dramatische Zuspitzung des Fachkräftemangels bezogen auf die MTRA hat zu einer } \\
\text { engen Zusammenarbeit aller radiologischen Fachgesellschaften seitens der MTRA (VMTB, VMTRO, } \\
\text { AG-MTM) und des DVTA geführt } \\
\text { - Treffen der Vorstände in Regensburg Anfang Januar unter Federführung des VMTB } \\
\text { - Treffen der Vorstände mit Erarbeitung strategischer Maßnahmen unter Federführung des DVTA }\end{array}$ \\
\hline
\end{tabular}


Tab. 2 (Fortsetzung)

\section{Arbeitsgemeinschaft, Zusammenkünfte, Aktivitäten im Berichtszeitraum und Planungen \\ Vorsitz}

Vorhaben $\quad$ - Vorbereitung und Organisation der MTRA-Tagung im Rahmen der DGN-jahrestagung 2020 in Leipzig

- gemeinsame Stellungnahme mit den Verbänden VMTB, VMTRO und DVTA zum Thema „MFA im Berufsfeld von MTRA“

\begin{tabular}{|c|c|c|}
\hline \multirow{3}{*}{$\begin{array}{l}\text { Neuronuklearmedizin } \\
\text { Prof. K.-J. Langen }\end{array}$} & Veranstaltungen & - Jahreshauptsitzung während der DGN-Jahrestagung, 04. April 2019, Bremen \\
\hline & Aktivitäten & $\begin{array}{l}\text { - Leitlinien } \\
\text { - die S1-Leitlinie für „SPECT-Untersuchungen mit dem }{ }^{123} \text {-markierten Dopamintransporter-Liganden } \\
\text { FP-CIT (DaTSCANTM)“ ist im Februar } 2019 \text { erschienen: Nuklearmedizin } 2019 \text { Feb; 58(1): 5-16 } \\
\text { - die S1-Leitlinie für „PET-Untersuchungen von Patienten mit Hirntumoren“ liegt dem DGN-Vorstand } \\
\text { seit Oktober } 2019 \text { zur weiteren Bearbeitung vor } \\
\text { - die S1-Leitlinie „Hirnperfusions-SPECT mit Tc-99m-Radiopharmaka“ liegt dem DGN-Vorstand zur } \\
\text { weiteren Bearbeitung vor }\end{array}$ \\
\hline & Vorhaben & $\begin{array}{l}\text { - Publikation der S1-Leitlinie der Deutschen Gesellschaft für Nuklearmedizin für „PET-Untersuchungen } \\
\text { von Patienten mit Hirntumoren“ in der Zeitschrift Nuklearmedizin } 2020\end{array}$ \\
\hline \multirow{3}{*}{$\begin{array}{l}\text { Pädiatrische } \\
\text { Nuklearmedizin } \\
\text { Prof. C. Franzius }\end{array}$} & Veranstaltungen & - Arbeitsgruppensitzung, 05. April 2019, Bremen \\
\hline & Aktivitäten & 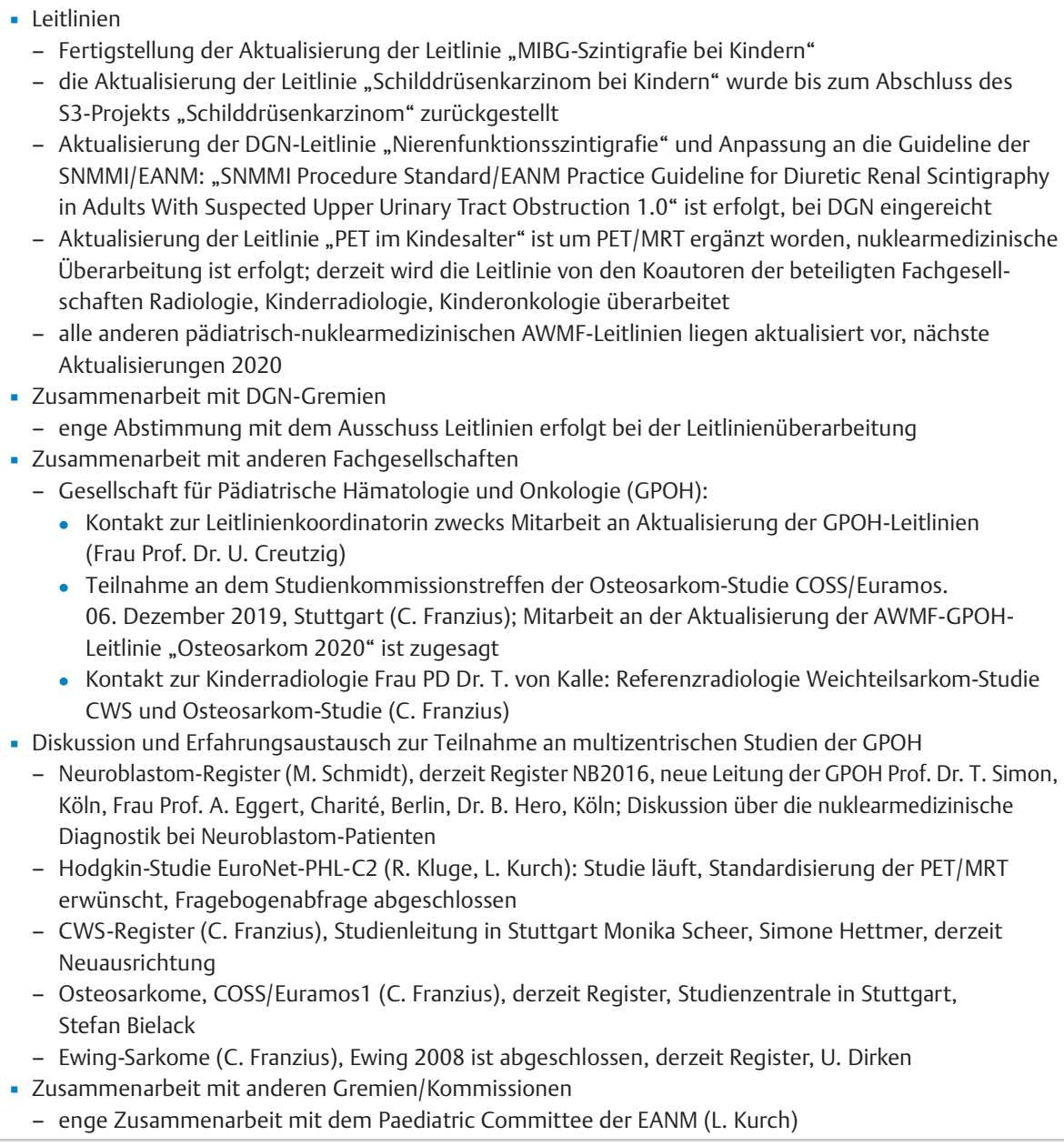 \\
\hline & Vorhaben & $\begin{array}{l}\text { " Abschluss der Aktualisierung der o. g. Leitlinien „PET im Kindesalter“ und Aktualisierung der Leitlinie } \\
\text { „Knochenszintigrafie bei Kindern“ für } 2020 \text { vorgesehen }\end{array}$ \\
\hline $\begin{array}{l}\text { Radiochemie und } \\
\text { Radiopharmazie } \\
\text { Prof. K. Kopka }\end{array}$ & Veranstaltungen & $\begin{array}{l}\text { - 27. Jahrestagung der AGRR (AGRR2019) (178 Teilnehmer), 12.-14. September 2019, Pamhagen, } \\
\text { Österreich } \\
\text { - 27. Mitgliederversammlung, 14. September 2019, Pamhagen } \\
\text { - } 60 . \text { Komitee-Sitzung, 12. September 2019, Pamhagen } \\
\text { - 59. Komitee-Sitzung, 04. April 2018, Bremen } \\
\text { - 58. Komitee-Sitzung, 30. März 2018, Pamhagen }\end{array}$ \\
\hline
\end{tabular}


Tab. 2 (Fortsetzung)

\title{
Arbeitsgemeinschaft, Zusammenkünfte, Aktivitäten im Berichtszeitraum und Planungen
} Vorsitz

\author{
Aktivitäten \\ - Leitlinien \\ - Leitlinien der AGRR gültig seit 25. September 2009 \\ - Fort- und Weiterbildungsaktivitäten \\ - Leitlinien der AGRR gültig seit 25. September 2009 \\ - Die Rolle der sachkundigen Person (Mag. pharm. A. Kraßnigg, Bundesamt für Sicherheit im \\ Gesundheitswesen, Österreich) \\ - Das Europäische Arzneibuch (Dr. E. Pel, European Pharmacopoeia Department, EDQM) \\ - Plenarvorträge im Rahmen der 27. AGRR-Tagung als Bestandteil des Programms \\ - Open Innovation Strategie (Prof.in Dr.in M. Poetz, Wien) \\ - Biomarkerentwicklung für molekulare Bildgebung mittels „Big Data“ (Dr. G. Egger, Wien) \\ - Fluoro-Bernd vs. Metal-Tom. Eine Unterhaltung (Prof. Dr. T. L. Mindt, Wien; Prof. Dr. B. Neumaier, \\ Jülich/Köln) \\ - Fachgruppe Nuklearchemie der GDCh (Beirat-Vertreter Prof. B. Neumaier) \\ - Postgraduate Certificate Course in Radiopharmaceutical Chemistry/Radiopharmacy (EANM) \\ - Beteiligung an DGN-Veranstaltungen oder anderen Veranstaltungen \\ - Tagung des DGN-Ausschusses „Hochschulfragen“, 22./23. November 2019, Berlin \\ - Vortrags- und Posterprogramm im Rahmen des $31^{\text {st }}$ Annual Congress of the European Association \\ of Nuclear Medicine (EANM'19), 12.-16. Oktober 2019, Barcelona, Spanien \\ - Vortrags- und Posterprogramm im Rahmen der Jahrestagung der Deutschen Pharmazeutischen \\ Gesellschaft (DPhG), 01.-04. September 2019, Heidelberg \\ - Vortrags- und Posterprogramm im Rahmen des $23^{\text {rd }}$ International Symposium on Radiopharmaceutical \\ Sciences (23rd ISRS), 26.-31. Mai 2019, Bejing, China \\ - Vortrags- und Posterprogramm im Rahmen des $66^{\text {th }}$ Annual Meeting der Society of Nuclear Medicine \\ and Molecular Imaging (SNMMI), 22.-25. Juni 2019, Anaheim, CA, USA \\ - Vortrags- und Posterprogramm im Rahmen der Jahrestagung der DGN (NuklearMedizin2019), \\ 03.-06. April 2019, Bremen \\ - $5^{\text {th }}$ Theranostics World Congress 2019, 01.-03. März 2019, Jeju, Korea \\ - eigene Veranstaltungen \\ - 27. Jahrestagung der AGRR (AGRR2019), 12.-14. September 2019, Pamhagen, Österreich \\ - fünfter AGRR-GMP-Workshop, 12. September 2019, Pamhagen (GMP-Beauftragter Dr. Christoph \\ Solbach, Ulm) \\ - Zusammenarbeit mit DGN-Gremien \\ - DGN-Ausschuss Hochschulfragen \\ - DGN-Ausschuss Radiopharmaka \\ - Zusammenarbeit mit anderen Fachgesellschaften \\ - Society of Radiopharmaceutical Sciences (SRS) \\ - European Symposium on Radiopharmacy and Radiopharmaceuticals (ESRR) \\ - European Association of Nuclear Medicine (EANM) \\ - Fachgruppe Nuklearchemie der Gesellschaft Deutscher Chemiker (GDCh) \\ - Deutsche Pharmazeutische Gesellschaft (DPhG) \\ - EANM/ESMIT Postgraduate Course Certificate of Advanced Studies (CAS) in Radiopharmaceutical \\ Chemistry/Radiopharmacy \\ - Österreichische Gesellschaft für Nuklearmedizin und Molekulare Bildgebung (OGNMB) \\ - Bündnis zur Förderung der Radiopharmazeutischen Wissenschaften (BüFraWi) \\ - Zusammenarbeit mit anderen Gremien/Kommissionen \\ - EANM Radiopharmacy Committee (Prof. Dr. M. Patt, Leipzig, Vorsitz; Mitglied seit 01. Januar 2015) \\ - EANM Drug Development Committee (Dr. W. Deuther-Conrad, Leipzig, Mitglied seit 01. Januar 2016) \\ - EANM Board Member (Assoc.-Prof. Dr. W. Wadsak; Vorstandsmitglied seit 01. Januar 2019) \\ - Zentralstelle der Länder für Gesundheitsschutz bei Arzneimitteln und Medizinprodukten (ZLG), \\ EFG (Expertenfachgruppe) 12 - Radiopharmaka (Leitung: Dr. M. Karmann), Klärung von aktuellen \\ Fragen des GMP/Arzneimittelrechts \\ - European Directorate for the Quality of Medicines (edqm) - Expertengruppe 14 der Europäischen \\ Arzneibuchkommission \\ - BfArM - AG Radiopharmaka
}


- Tab. 2 (Fortsetzung)

\section{Arbeitsgemeinschaft, Zusammenkünfte, Aktivitäten im Berichtszeitraum und Planungen} Vorsitz

\begin{tabular}{|c|c|c|}
\hline & & $\begin{array}{l}\text { - anderweitige Aktivitäten (z. B. Studien, Publikationen) } \\
\text { - AGRR-Leitlinien } \\
\text { - Deutsches Konsortium für Translationale Krebsforschung (DKTK): Radiopharmazie für die klinische } \\
\text { Prüfung der Phasen I und II mit dem Radiopharmakon } 68 \text { Ga-PSMA-11 } \\
\text { - Vergabe des AGRR-Preises für die besten Vorträge } 2019 \text { (Nachwuchswissenschaftler): } \\
\text { - Doktorand J. Pfister (Medizinische Universität Innsbruck, Klinik für Nuklearmedizin AG Clemens } \\
\text { Decristoforo). „Choose your label wisely: hybrid imaging of Aspergillus fumigatus infections using } \\
{ }^{68} \text { Ga-labelled, fluorescent siderophores“ } \\
\text { - Doktorandin A. S. Cankaya (Johannes-Gutenberg-Universität Mainz HZDR, Institut für Kernchemie, } \\
\text { AG Frank Rösch). „ " }{ }^{18} \text { F-markierte Cysteinprotease-Inhibitoren mit Vinylsulfonstruktur zur } \\
\text { Untersuchung von Tumoren mit erhöhter Cathepsin-Expression” } \\
\text { - Doktorand T. Kanagasundaram (DKFZ Heidelberg, Abteilung Radiopharmazeutische Chemie, } \\
\text { AG K. Kopka). „Synthesis of radiolabeled Si-rhodamines for bimodal imaging” }\end{array}$ \\
\hline & Vorhaben & $\begin{array}{l}\text { - 28. Jahrestagung der AGRR (AGRR2020), 12.-14. September 2020, Leipzig, Wittenberg (M. Patt, P. Brust) } \\
\text { - 29. Jahrestagung der AGRR (AGRR2021), 2021, Tübingen (G. Reischl) } \\
\text { - 30. Jahrestagung der AGRR (AGRR2022), angefragt: Hannover } \\
\text { - 31. Jahrestagung der AGRR (AGRR2023), in Planung }\end{array}$ \\
\hline \multirow{3}{*}{$\begin{array}{l}\text { GAPTN } \\
\text { Prof. L. Geworski } \\
\text { Prof. W. Burchert }\end{array}$} & Veranstaltungen & $\begin{array}{l}\text { - Arbeitsgruppentreffen auf der DGN 2019, 05. April 2019, Bremen } \\
\text { - 17. GAPTN-Jahrestreffen (2-tägig), 08.-09. Februar 2019, Medizinische Hochschule Hannover, Hannover }\end{array}$ \\
\hline & Aktivitäten & $\begin{array}{l}\text { - Fort- und Weiterbildungsaktivitäten } \\
\text { - s. o.; anerkannt DGMP } \\
\text { - Beteiligung an DGN-Veranstaltungen oder anderen Veranstaltungen } \\
\text { - eigene Veranstaltungen } \\
\text { - s. o. } \\
\text { - Zusammenarbeit mit DGN-Gremien } \\
\text { - Ausschuss Strahlenschutz } \\
\text { - Ausschuss Medizinische Physik } \\
\text { - Zusammenarbeit mit anderen Gremien/Kommissionen } \\
\text { - NAR-DIN } \\
\text { - SSK A2, A4 } \\
\text { - BfS } \\
\text { - Aufsichtsbehörden }\end{array}$ \\
\hline & Vorhaben & $\begin{array}{l}\text { - Treffen auf der DGN-Jahrestagung 2020, Leipzig } \\
\text { - 18. GAPTN-Jahrestagung, 06.-07. März 2020, Medizinische Hochschule Hannover, Hannover }\end{array}$ \\
\hline
\end{tabular}

